\title{
L'enseignement du catalan en tant que langue régionale en France - État des lieux 2009
}

Mary Sanchiz et Luc Bonet

\section{(2) OpenEdition}

\section{Journals}

Édition électronique

URL : http://journals.openedition.org/trema/956

DOI : 10.4000/trema.956

ISSN : 2107-0997

Éditeur

Faculté d'Éducation de l'université de Montpellier

Édition imprimée

Date de publication : 1 septembre 2009

ISSN : 1167-315X

Référence électronique

Mary Sanchiz et Luc Bonet, « L'enseignement du catalan en tant que langue régionale en France État des lieux 2009 », Tréma [En ligne], 31 | 2009, mis en ligne le 24 février 2010, consulté le 19 avril 2019. URL : http://journals.openedition.org/trema/956 ; DOI : 10.4000/trema.956

Ce document a été généré automatiquement le 19 avril 2019

Trema 


\title{
L'enseignement du catalan en tant que langue régionale en France - État des lieux 2009
}

\author{
Mary Sanchiz et Luc Bonet
}

1 Dans l'enseignement supérieur, le catalan est enseigné dans nombre d'universités françaises : à Bordeaux 3, Lyon 2, Grenoble 3 par exemple, pour non spécialistes seulement; à Montpellier 3, Paris 4 par exemple, comme une spécialisation du cursus d'espagnol; à Toulouse 2 pour les deux premières années de licence de catalan. Cependant, c'est dans le département des Pyrénées - Orientales que se concentrent à la fois la quasi - totalité des élèves et des professeurs enseignant spécifiquement le catalan, de la maternelle à la fin des études supérieures (l'université de Perpignan propose un cursus complet d'études catalanes) ${ }^{1}$.

2 C'est de cette constatation que nous partirons en ciblant notre analyse sur ce département et sans revenir sur un aperçu historique qui s'étend sur plus d'un demi - siècle depuis la loi DEIXONNE (MARTEL, 2007), qui a déjà été largement exploré par une somme d'articles et qui, de toute façon, est à peu

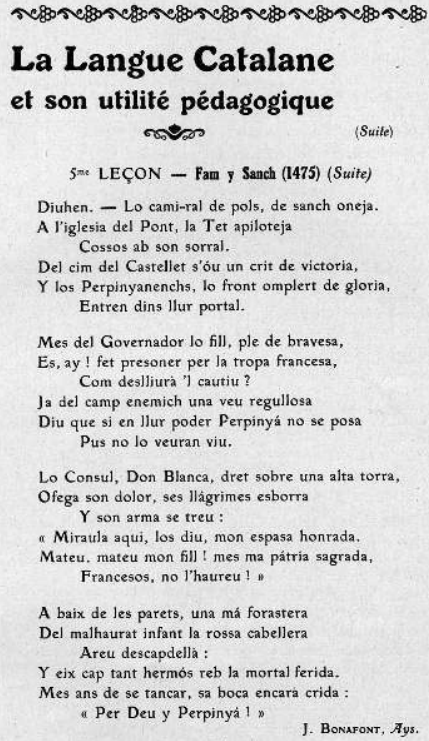


près le même pour toutes les langues régionales de France. Néanmoins, du point de vue de la sociolinguistique et de l'histoire, le catalan se distingue d'autres langues régionales de France. Jamais qualifié de "patois» même du temps de l'abbé Grégoire ou des "Hussards noirs de la République », la langue catalane au Nord des Pyrénées se nourrit aussi à partir de la Renaixença littéraire barcelonaise de la fin du XIXe siècle et surtout depuis le rétablissement des institutions propres à la Catalogne espagnole, à la fin des années soixante - dix, de la proximité des locuteurs du Sud des Pyrénées qui représentent toujours aujourd'hui l'essentiel des quelque 9 millions de catalanophones. Paradoxalement, le catalan n'a jamais été précurseur dans la revendication de l'enseignement des langues régionales en France, peut - être parce que la frontière politique avec l'Espagne a toujours été plus ou moins perméable (même sous la dictature franquiste) et le devient de plus en plus dans l'espace européen. Les échanges familiaux (particulièrement après l'exode de la Retirada (1939), économiques (entreprises multinationales de part et d'autre de la frontière : Mitjavila, Milles) et politiques (accords transfrontaliers d'échanges scolaires, de coopération (hôpital transfrontalier de Puigcerdà) gomment, chaque jour un peu plus, comme pour toutes les régions transfrontalières qui ont une unité linguistique (Pays basque, Alsace), les différences de niveau de vie et de régime politique.

Pourtant, dans les Pyrénées - Orientales, le catalan n'est plus, depuis la fin de la seconde guerre mondiale, la langue maternelle des enfants. Mais il est encore, pour beaucoup de familles d'origine rurale, la langue des arrière - grands - parents qui en font un usage diglossique alors qu'il est devenu, en particulier depuis les Jeux Olympiques de Barcelone en 1992, la langue du dynamisme économique outre - Pyrénées.

Pour tenter de dresser un état objectif de l'enseignement du catalan dans les Pyrénées Orientales, nous partirons donc de deux faits qui nous semblent significatifs dans les vingt dernières années :

1. La mise en place des premières sections bilingues à parité horaire depuis la maternelle à partir de 1993

2. La mise en place dès 2006 de programmes en fonction du Cadre européen commun de référence.

\section{La mise en place des sections bilingues depuis la maternelle}

5 La mise en place des sections bilingues depuis la maternelle est une date importante dans l'évolution didactique et pédagogique. En effet, auparavant, après les premières expériences dans le second degré, lors des années cinquante et soixante, et dès 1975, avec la participation militante du Grup Rossellonès d'Estudis Catalans, l'école primaire publique s'était ouverte aux premières expériences d'enseignement du catalan. À partir de 1976, l'école associative La Bressola, d'abord timidement, ensuite dans une vraie ligne ascendante d'augmentation constante des effectifs, offrit et offre toujours un enseignement du catalan par immersion totale précoce.

6 Ces écoles La Bressola, dont le rôle positif est évident dans le maintien de la tradition linguistique, entretiennent néanmoins l'idée d'un parallélisme séparateur entre la langue « officielle » et la langue "régionale ». Les élèves inscrits dans ce système linguistique cloisonnent manifestement l'apprentissage des deux langues, y compris au moment de 
l'apprentissage de la lecture. C'était d'ailleurs aussi la situation d'apprentissage de toutes les langues étrangères à l'école française avant la récente prise en compte du Cadre européen commun de référence: chaque système linguistique reste un espace fermé considéré comme une "matière" à part entière et génère peu de réflexion métalinguistique.

Depuis le début des années 1990, et surtout à partir de la circulaire de 1995 qui organise l'enseignement bilingue paritaire entre le français et la langue régionale, sur le modèle français - basque des écoles publiques pionnières des Pyrénées - Atlantiques, l'enseignement bilingue à parité horaire entre le français et le catalan, lancé de fait dès 1993 à l'école maternelle Jean - Amade de Perpignan, met en place des situations didactiques et pédagogiques qui vont faire évoluer la réflexion au - delà de la reproduction du modèle immersif de la Catalogne autonome, lui - même inspiré du système canadien.

8 En effet, le principe de la parité horaire revenant à de l'immersion partagée entre français et catalan induit indubitablement une approche comparative et rompt avec une tradition «d'exclusivisme culturel ( (MARTEL, 1999). Les deux langues perdent leur aire propre d'apprentissage pour entrer dans un système de liens et de comparaisons qui change fondamentalement le regard porté sur leur apprentissage simultané. Nourrie par les observations faites par ailleurs dans les lycées français à l'étranger (DUVERGER, 2007, p. 21), cette approche contrastive et comparative permet à l'élève des transferts incessants d'une langue à l'autre et d'autant plus féconds qu'ils interviennent dans une sorte de giron commun, celui de la langue mère latine.

9 Le pédagogue occitano - catalan Louis PASTRE, instituteur à Perpignan au début du siècle passé avait déjà réfléchi à cette question en proposant une méthode mixte allant au - delà du syncrétisme didactique entre français et langue régionale des précurseurs occitans de la pédagogie régionaliste (BONET, 2007). Aujourd'hui, pour la première fois, il s'agit d'une posture officielle. Ainsi se construisent, en même temps, les apprentissages de la lecture et de l'écriture dans les deux langues et l'objectif est que tous les élèves aient acquis à l'entrée au lycée et grâce à une réflexion métalinguistique continue, non seulement des compétences identiques dans chaque langue, mais encore des capacités de transfert réutilisables dans tous les domaines et a fortiori dans le domaine de l'apprentissage d'autres langues (bi - plurilinguisme) (DUVERGER, 2005 ; BONET, 2007). La première promotion d'élèves bilingues depuis la maternelle de l'école Joan Amade en 1993 (BONET, 1997) a passé le baccalauréat en 2006 confirmant les hypothèses didactiques. Mais trop peu d'élèves bénéficient encore aujourd'hui de ce cursus complet pour pouvoir livrer de vraies statistiques.

10 L'arrêté du 12 mai 2003 paru consécutivement à l'annulation par le conseil d'état en 2002 des textes traitant conjointement de l'enseignement par immersion totale et de l'enseignement à parité horaire précise qu' "aucune discipline autre que la langue régionale ne peut être enseignée exclusivement en langue régionale » et a finalement permis, indirectement, une évolution favorable des classes bilingues publiques vers une approche linguistique décloisonnée plus "productive », même si des statistiques précises à ce sujet font encore défaut. Une seule école publique fonctionne encore en immersion totale jusqu'au cycle 2 dans les Pyrénées - Orientales : l'école ARRELS intégrée à l'école publique avant le décret en question.

11 Cependant, il manque encore bien des éléments du puzzle: les écoles La Bressola ne conduisent qu'une partie restreinte de leurs élèves jusqu’à la fin du collège (10 élèves de 
troisième à la rentrée 2008 pour un total général de 658 élèves) et la parité horaire, si elle est effective et bien assurée dans les classes primaires, ne peut guère se mettre vraiment en place au collège et au lycée, sauf exceptions, au - delà d'une ou deux disciplines non linguistiques, faute d'enseignants qualifiés, de surcharge des emplois du temps ou de moyens horaires. Car, si une classe complète d'élèves bilingues ne demande certes pas de moyens supplémentaires (le professeur assure le même nombre d'heures, qu'il enseigne en catalan ou en français), une classe incomplète doit forcément être dédoublée, entraînant une perte non négligeable d'heures dans la dotation globale de l'établissement.

De la théorie à la pratique, chaque établissement s'adapte donc en fonction de ses possibilités et la situation est loin d'être uniforme. Néanmoins, les effectifs sont en augmentation régulière depuis la création des classes bilingues, preuve qu'elles correspondent à un réel besoin et à une vraie demande.

\section{Evolution de l'enseignement bilingue dans les PO}

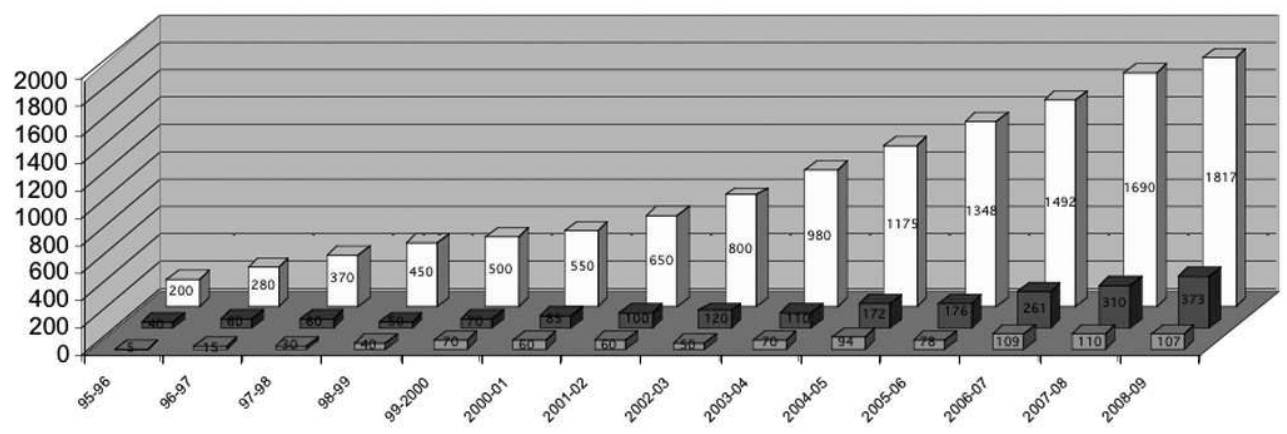

Pourtant, les effectifs accusent deux paliers critiques dans un cursus complet: la déperdition de l'entrée en sixième et celle de l'entrée en seconde.

C'est évidemment la déperdition de l'entrée en sixième qui est la plus grave. Les raisons déjà invoquées de surcharge des emplois du temps y sont sans doute pour quelque chose, mais d'autres facteurs interviennent.

Le premier est que l'imaginaire collectif des parents reste encore aujourd'hui dans une perspective de survie de la langue. Les parents interrogés (enquêtes pour les ouvertures de classes) estiment donc avoir fait en quelque sorte leur "devoir de transmission" puisque les enfants sont effectivement bilingues en fin de CM2. Ce que beaucoup de parents n'ont pas encore suffisamment intégré, et que le Cadre Européen Commun de Référence met en évidence si on veut bien s'y reporter aussi pour la langue maternelle, c'est que la compétence linguistique est également fonction de la maturation conceptuelle. Les enfants sont donc bilingues, mais pour des compétences de fin d'école primaire (semblables à celle du français), c'est - à - dire par exemple sans grande capacité d'abstraction ou d'argumentation. Il nous reste donc un effort à faire (peut - être d'information) pour convaincre que l'apprentissage d'une langue, quelle qu'elle soit, n'est efficace dans le monde du travail des adultes que si elle atteint un degré élevé de compétences conceptuelles. De la même façon, le bénéfice de la valeur ajoutée cognitive que génère un enseignement de disciplines non linguistiques dans deux langues ne se révèle vraiment que sur la durée d'un cursus scolaire complet. Ces remarques sont évidemment encore plus vraies au lycée.

Le second est la concurrence des options, au collège comme au lycée : curieusement, cela concerne peu l'anglais, puisqu'il est la langue vivante étrangère la plus largement choisie 
en LV1 ou en LV2. Mais la concurrence se met vite en place avec les autres langues vivantes étrangères (en particulier pour les classes bi - langues et les classes européennes), avec le latin au collège, et toutes les options de détermination de la classe de seconde au lycée. Théoriquement, toutes les options sont ouvertes aux classes bilingues, mais la réalité de l'emploi du temps ou d'une saturation d'heures de cours rend les choses moins évidentes sur le terrain. C'est la raison pour laquelle Monsieur le Recteur favorise l'implantation de parcours latino - romans quand ils peuvent être mis en place car ils permettent un ajustement plus efficace des emplois du temps et favorisent les apprentissages communs. Ce travail commun demande, en revanche une grande concertation entre les professeurs et reste parfois tributaire des aléas des mutations des enseignants.

À côté de l'enseignement bilingue existent évidemment l'apprentissage d'initiation à l'école primaire avec une langue vivante étrangère, de langue et culture régionale au collège (d'une à deux heures par semaine et par niveau suivant les possibilités), de LV2 / LV3 au collège et au lycée, y compris dans l'enseignement privé confessionnel.

\section{EFFECTIFS 2008-2009}

\begin{tabular}{|c|c|c|c|c|c|}
\hline & \multicolumn{2}{|c|}{ Enseignem ent public } & Enseigne & \multicolumn{2}{|c|}{ ment privé } \\
\hline & & & Confessionnel & \multicolumn{2}{|c|}{ Associatif } \\
\hline \multirow[t]{2}{*}{ CATALAN } & \multirow{2}{*}{$\begin{array}{c}\text { Facult./LV2/LV3/ } \\
\text { option }\end{array}$} & \multirow[t]{2}{*}{ Bilingues } & \multirow[t]{2}{*}{ Facult. et LV } & \multicolumn{2}{|c|}{ Immersion } \\
\hline & & & & Contrat & H. Contrat \\
\hline Maternelles & & 559 & & & \\
\hline Primaire & & 1234 & 166 & & \\
\hline $\begin{array}{l}=1^{\circ} \\
\text { DEGRE }\end{array}$ & $\begin{array}{c}6357 \text { (initiation) + } \\
1670(\mathrm{LV})= \\
8027\end{array}$ & 1793 & 166 & 482 & 97 \\
\hline Collèges & 1564 & 373 & 172 & 46 & 58 \\
\hline Lycées & 292 & 107 & 118 & & \\
\hline BP & 90 & & & & \\
\hline BTS & 35 & & & & \\
\hline$=2^{\circ}$ DEGRE & 1951 & 480 & 290 & & \\
\hline \multirow{2}{*}{$\begin{array}{l}\text { Total } 1^{\circ}-2^{\circ} \\
\text { degrés }\end{array}$} & \multirow[b]{2}{*}{10008} & \multirow[b]{2}{*}{2273} & \multirow[b]{2}{*}{456} & 528 & 155 \\
\hline & & & & 6 & 83 \\
\hline TOTAL GL & \multicolumn{2}{|c|}{12281} & \multicolumn{3}{|c|}{1139} \\
\hline
\end{tabular}

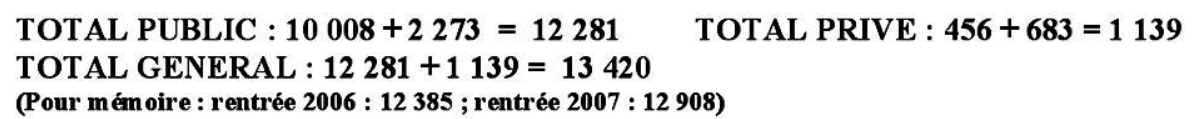

18 Sur un total d'élèves à l'école primaire publique de 38043 à la rentrée 2008, 25,8 \% suivent un enseignement de catalan, 4,7 \% suivent un enseignement bilingue.

Dans l'enseignement public, le personnel enseignant est constitué de 46 professeurs certifiés de catalan (places attribuées au CAPES depuis 1992) qui n'enseignent pas tous en classe bilingue et d'environ 75 professeurs d'école (qui enseignent en classe bilingue). 


\section{La mise en place de programmes en fonction du cadre européen de compétences}

20 L'adoption par la France du Cadre européen commun de référence a généré une réécriture générale des programmes de langues vivantes, y compris en 2006 (publication 2007) de ceux des langues régionales, pour la première fois de leur histoire. En effet, jusqu'à cette date, il existait un programme allégé pour les écoles primaires et les grandes lignes d'un programme pour les lycées. Mais dans la mesure où rien n'avait été publié pour les collèges, la cohérence de l'ensemble du cursus ne reposait que sur la concertation entre professeurs. La réécriture générale de l'ensemble en fonction des niveaux de compétences du Cadre européen (A1 - A2, déjà publiés, et B1 - B - 2, en cours de publication) a permis non seulement une harmonisation des niveaux de compétence de toutes les langues vivantes, quelles qu'elles soient, étrangères ou régionales, mais aussi une harmonisation des objectifs d'enseignement et des pratiques (BONET, 2007).

21 De cette façon, une approche actionnelle des apprentissages est en train de se généraliser. En effet, le catalan, surtout dans les zones rurales est encore aujourd'hui compris par une grande majorité de la population autochtone, et parlé couramment par la génération de 60 - 80 ans. C'est dire combien il est facile de construire en classe des projets touchant directement une partie de la population en catalan, voire l'ensemble si on veut bien en faire une édition bilingue.

Un exemple: la municipalité de $\mathrm{S}$. a demandé aux élèves du collège de réaliser un prospectus à distribuer pour annoncer les festivités de la Saint - Jean. Les élèves de catalan ont ainsi fait des recherches (origine des traditions), présenté un résumé bilingue de leurs trouvailles, annoncé le programme et mis en forme le tout avec un logiciel de P.A.O. Ils ont évidemment ensuite participé aux festivités. Si nous citons cet exemple, c'est pour montrer qu'une approche actionnelle ne demande pas forcément de grands moyens pour s'insérer dans une vraie pratique sociale de référence. En catalan, comme dans les autres langues régionales, elle est encore plus évidente que dans les langues vivantes étrangères où les professeurs ont souvent recours aux jeux de rôle, faute d'un public véritablement réceptif.

La réécriture des programmes dans le cadre européen de référence a également induit une réflexion de l'équipe de rédaction sur la variante dialectale septentrionale. Pour le catalan, l'unification de l'orthographe, la dépuration et l'enrichissement du lexique, la fixation de la grammaire normative furent l'œuvre de Pompeu FABRA et de l'Institut d'Estudis Catalans de Barcelone entre 1913 et 1919 ; le Diccionari general de la llengua catalana ne fut publié qu'en 1932 à cause des vicissitudes politiques espagnoles. Ce cadre normatif est pleinement assumé sur le territoire septentrional du domaine linguistique catalan constitué, à l'exception des Fenouillèdes de langue d'oc, par l'essentiel du département des Pyrénées - Orientales. Cependant, cette norme d'une langue catalane naturellement très unitaire n'exclut pas les variantes dialectales du nord du domaine catalan, parlées dans le département des Pyrénées - Orientales ; l'enjeu est donc important.

Enfin, les programmes ainsi réécrits ont permis d'intégrer les classes bilingues à l'ensemble. Puisqu'il s'agit désormais d'un niveau général de compétences, seul le niveau attendu en fin de cycle sépare désormais l'initiation, les LV2 - 3 et les classes bilingues. Il faut cependant être attentif au fait que ce niveau de compétences attendu n'est pas 
seulement linguistique. Il vise aussi un degré culturel et un degré de conceptualisation des apprentissages. C'est pourquoi, ce niveau a été fixé nationalement à A2 pour la fin des classes bilingues de l'école primaire, B2 pour la fin du collège et $\mathrm{C} 1$ pour la fin du lycée, même si linguistiquement certains de ces élèves sont nettement au - dessus. Cette échelle de niveaux de compétence des classes bilingues correspond également aux attentes pour la langue catalane des autres systèmes éducatifs concernés, de Catalogne espagnole et d'Andorre en particulier.

\section{Les liens avec les autres disciplines}

Sans parler des disciplines non - linguistiques des classes bilingues qui suivent les programmes nationaux des dites disciplines, mais en rajoutant, chaque fois que faire se peut des exemples locaux, suivant en cela l'insertion du patrimoine dans le socle commun, les programmes de catalan ont été réécrits en parallèle avec les programmes de lettres, lesquels s'alignaient déjà sur ceux d'histoire - géographie pour les grandes scansions de l'histoire de l'humanité. Cette volonté d'alignement à la fois culturel (chronologie historique pour contextualiser les œuvres littéraires qui portent forcément en elles des échos de la période pendant laquelle elles ont été écrites) et conceptuel (apprentissage du discours narratif et descriptif à l'école primaire, avec simplement quelques approches de l'explicatif et de l'argumentatif, puis approfondissement du narratif et de l'explicatif au collège en rajoutant progressivement l'argumentatif) facilite évidemment les « ponts » avec les autres disciplines.

Par ailleurs, toute mise en pratique de liens entre la langue - mère latine et les langues romanes ne peut que faciliter les apprentissages grammaticaux et lexicaux. C'est pourquoi, depuis plusieurs années maintenant, nous l'avons déjà mentionné supra, des parcours latino - romans tentent de mettre en cohérence les programmes de latin et les programmes de catalan. Il est évident qu'une telle réflexion concerne aussi les autres langues romanes, régionales ou nationales. Il y a là un grand chantier qui s'ouvre pour les années à venir.

Un pas important dans ce sens a été fait en 2006 dans l'académie de Montpellier par la promulgation par le Recteur Christian NIQUE d'un «Plan de développement des langues régionales » et par la publication prévue dans ce plan, sous sa direction, de trois ouvrages distribués à l'ensemble des établissements et destinés à favoriser l'approche des deux langues régionales de l'académie (le catalan et l'occitan) par les enseignants "non spécialistes ». Il s'agit d'un précis de langue, d'un précis d'histoire et d'une anthologie littéraire. Des pistes de travail en ligne sur le site parachèvent le travail (http ://www.acmontpellier.fr).

\section{Perspectives d'avenir}

\section{IV.1. La demande scolaire}

Malgré ces avancées significatives, la demande est nettement supérieure à l'offre et souvent difficile à satisfaire en fonction des moyens limités : neuf postes pourvus en 2008 au CRPE et un poste au CAPES : c'est moins que les besoins avérés en particulier en matière de remplacements. 
29 En l'absence d'enquêtes générales très récentes en la matière (beaucoup d'enquêtes aujourd'hui ne concernent que les secteurs d'implantation de projets de classes bilingues), nous citerons néanmoins deux chiffres :

- 1995 (enquête de l'Inspection académique des P.O.) 37,6 \% des parents sont favorables au bilinguisme à la maternelle (BECAT, 2000, p. 29)

- 1998 (seconde enquête de « Média Pluriel Méditerranée ») 62 \% des parents sont favorables à un enseignement du catalan en général (initiation et bilinguisme inclus) à l'école primaire (PUIG - MORENO, 2007, p. 27).

Par ailleurs, aujourd'hui, cette demande semble évoluer très vite. En effet, les lycées professionnels incluent, de plus en plus, l'initiation à la langue catalane dans leurs cursus et envisagent des stages d'entreprise ultra - frontaliers. Céret et Prades ont commencé. Ces deux établissements vont développer leurs projets l'an prochain. Le lycée hôtelier Léon Blum, qui a toujours eu quelques élèves de BTS (35 cette année) a également un projet important pour ses baccalauréats professionnels.

31 Aussi bien dans le secteur de l'hôtellerie que dans le tertiaire et même dans l'ensemble des métiers qui touchent de près ou de loin au bâtiment, c'est évidemment le développement de la plateforme logistique européenne Saint - Charles pour les fruits et légumes, l'extension de quelques entreprises des Pyrénées - Orientales en Catalogne du Sud (Mitjavila, Milles...) , la construction de résidences secondaires sur le haut - plateau de Cerdagne pour la bourgeoisie barcelonaise, et l'arrivée prochaine à Perpignan de la ligne ferroviaire à grande vitesse en provenance de Barcelone qui ont entraîné cette mutation.

32 Ces demandes sociales ont d'abord concerné le GRETA (secteur de Font - Romeu en particulier) et se convertissent aujourd'hui en demandes scolaires. Même si ces secteurs économiques sont moins florissants depuis quelque temps déjà et sont aujourd'hui frappés par la crise, la demande scolaire reste dans une phase d'accroissement.

\section{IV.2. Les moyens humains}

33 Trois problèmes importants sont aujourd'hui particulièrement épineux : le manque de professeurs d'école ou d'intervenants extérieurs prenant en charge les cours d'initiation à l'école primaire, le manque de professeurs de disciplines non - linguistiques compétents pour enseigner en catalan et le manque de professeurs d'école bilingues.

Le nombre de catalanophones natifs susceptibles d'intervenir en milieu scolaire ayant fortement diminué depuis quelques années (départ à la retraite d'enseignants nés pendant ou juste après la guerre, recul général de la langue dans les villes et les zones rurales), l'as - sociation APLEC, chargée par convention avec l'Inspection d'académie d'intervenir quand l'Éducation nationale n'a pas le personnel nécessaire, rencontre elle aussi des difficultés. Le recrutement auprès des étudiants de l'université (Institut Franco Catalan Transfrontalier) est insuffisant tout comme, c'est une évidence, la formation didactique et pédagogique qu'ils peuvent recevoir malgré la bonne volonté de tous les acteurs.

Les intervenants en disciplines non linguistiques (DUVERGER, 2007, p. 81) sont de deux sortes : les certifiés de catalan qui ont passé une épreuve de seconde valence en histoire géographie (soit une épreuve du CAPES correspondant) et ceux, professeurs certifiés (ou agrégés) d'une autre discipline, dépendant donc de leur propre inspection pédagogique 
régionale, mais possédant une compétence en langue catalane garantie par l'inspection pédagogique régionale de catalan. Leur appartenance à leur corps d'origine souligne leur compétence dans la matière enseignée mais leur niveau linguistique et l'adaptation au milieu local fera, dès l'année prochaine, faute de manuels spécifiques, l'objet de la formation continue assurée par la Délégation Académique de Formation du Personnel de l'Education Nationale, seule compétente en la matière.

Le manque de professeurs d'école bilingues est néanmoins aujourd'hui le problème majeur. Car il ne pourra y avoir d'extension des cohortes d'élèves catalanophones au collège et au lycée que si l'école peut ouvrir des sections bilingues ou des initiations à la langue de qualité. Or la petitesse de l'aire de recrutement (le seul département des Pyrénées - Orientales) limite le vivier malgré l'investissement important de l'IUFM (antenne de Perpignan) dans la préparation et le suivi des concours d'enseignement (CAPES et CRPE spécifiques). Certes, et fort heureusement, des étudiants viennent parfois d'autres universités ou de la Catalogne du sud pour passer les concours spécifiques, le CAPES de catalan en particulier, mais cet apport aujourd'hui reste insuffisant car tout enseignant, professeur d'école de surcroît, doit être compétent dans les deux langues.

\section{IV.3. Les moyens financiers}

En l'absence de signature de toute convention entre l'académie et la région et / ou le département des Pyrénées - Orientales, les moyens en heures - poste et en heures supplémentaires sont entièrement supportés par l'Éducation nationale. La Région, le Département et les municipalités interviennent aujourd'hui uniquement sous forme de subventions importantes à l'association APLEC sus - citée pour les intervenants extérieurs, aux établissements privés, et au pôle de ressources du Centre Départemental de Documentation Pédagogique pour l'édition et l'animation pédagogique sous la double responsabilité du directeur du centre et de l'inspection pédagogique régionale.

\section{Conclusion}

La situation du catalan a favorablement évolué ces dix dernières années, comme en témoignent les effectifs en constante progression. Les élèves qui fréquentent aujourd'hui les classes bilingues ne sont pas forcément d'origine catalanophone, ni francophone d'ailleurs. Le choix d'une scolarité bilingue n'est plus teinté de militantisme politique mais est, de plus en plus fréquemment, motivé par un souci d'intégration culturelle à la région et d'intercompréhension de part et d'autre de la frontière (perspectives économiques). Ainsi, le regard porté sur la connaissance de la langue et de la culture catalanes, senties comme un " plus » dans le cursus scolaire des élèves et non comme une scorie du passé, a permis à l'enseignement de se «normaliser » chaque jour un peu plus. Mais les textes épars qui le régissent mériteraient d'être réunis et élargis dans le cadre d'une loi qui prendrait véritablement en compte le niveau réel des élèves: au baccalauréat les élèves bilingues comme les autres composent en LV2 ou sont interrogés à l'oral en LV3 selon leur choix. La discipline non - linguistique prise en compte est seulement l'histoire - géographie (rédaction en catalan de la réponse au sujet général). Au diplôme national du brevet, il en est de même pour l'histoire - géographie et, pour l'année 2010, le niveau A2 de catalan, en complément du niveau A2 en langue vivante étrangère, sera pris en compte (arrêté du 9 - 7 - 2009, J.O. du 25 - 7 - 2009). 


\section{BIBLIOGRAPHIE}

BECAT I RAJAUT, Joan (2000). La situació del català a França, Aspectes jurídics i docents i estudis sobre la matèria. Barcelona : Institut d'Estudis Catalans.

BONET, Lluc (1997). El català a l'escola a la Catalunya del Nord, Revista de Catalunya, n¹19, Barcelona.

BONET, L. (2007). Les sections bilingues français - langue régionale et le cadre européen : nouvelles perspectives didactiques, Tréma, « Plurilinguisme et enseignement », n² 28.

BONET, L. (2007). Louis PASTRE précurseur de l'enseignement intégré du catalan et du français à l'école publique, LIEUTARD, H., VERNY, M. - J, (coordonné par), L'école française et les langues régionales, XIXe - XXe siècles, E.T.O.I.L.L., Presses universitaires de la Méditerranée.

DUVERGER, Jean (coordonné par) (2000). Le français dans le monde, « Actualité de l'enseignement bilingue ", Janvier 2000.

DUVERGER, J. (2005). L'Enseignement en classe bilingue, Hachette (FLE).

DUVERGER, J. (2007), « Ecole élémentaire et enseignement des langues » et « Didactiser l'alternance des langues en cours de DNL », in revue Tréma, «Plurilinguisme et enseignement », N ${ }^{\circ} 28$.

MARTEL, Philippe (1999). Apprendre à parler en langue régionale pour devenir citoyen, Tréma, actes du colloque « Savoir, langages et citoyenneté », $\mathrm{n}^{\circ} 15-16$.

MARTEL, P. (2007). L'école française et l'occitan, Le sourd et le bègue, E.T.O.I.L.L., Presses universitaires de la Méditerranée.

NIQUE, Christian (sous la direction de...), SANCHIZ, Mary et TORREILLES, Claire (rédaction). (2006). Précis d'occitan et de catalan, SCEREN - Académie de Montpellier.

NIQUE, C. (sous la direction de...), SANCHIZ, Mary, et TORREILLES, Claire (rédaction). (2006). Petite anthologie des littératures occitane et catalane, SCEREN - Académie de Montpellier.

NIQUE, C. (sous la direction de...), LIMOUZIN, Jacques (rédaction). (2007). Précis d'histoire du Languedoc - Roussillon, SCEREN - Académie de Montpellier.

PUIG MORENO, Gentil. (2007). Dossier « Enquestes sociolingüístiques a la Catalunya Nord in Aïnes noves 1 , colllecció setelCAT, Premses Universitàries de Perpinyà.

\section{NOTES}

1. En France, dans le département des Pyrénées - Orientales, le catalan qui est enseigné de la maternelle à l'université est aussi une langue d'enseignement. Dans les écoles publiques, les classes bilingues fonctionnent selon la parité horaire hebdomadaire entre le français et la langue régionale. De plus, le catalan est la langue officielle de l'Andorre, état membre de l'ONU; en Espagne, elle est langue co - officielle (Catalogne, Baléares, Valence) ou reconnue (Aragon) ; en Italie, langue protégée (ville d'Alghero). Le catalan est une langue unitaire avec de faibles variations dialectales vivantes; la variante septentrionale (rossellonès) concerne pleinement le Roussillon, le Conflent, le Capcir et le bas Vallespir; le haut - Vallespir et la Cerdagne ont quelques caractéristiques divergentes. La langue catalane, disposant d'une académie centenaire ( Institut d'Estudis Catalans) qui fixe les normes de la langue standard, concerne un territoire peuplé 
de près de 13 millions d'européens dont 9 millions sont capables de la parler, tous au moins bilingues. Le catalan est enseigné dans 166 universités hors de son domaine linguistique, sur les cinq continents, en particulier en Europe mais aussi en Amérique; la langue catalane, très ouverte aux nouvelles technologies, dispose depuis 2006 du domaine internet « .cat » qui lui est spécifique. Le catalan est un cas unique parmi les langues minoritaires de par son statut, sa démographie, sa situation sociolinguistique, son bagage linguistique et sa tradition culturelle et littéraire (75 000 titres disponibles en langue catalane, plus de 10000 titres édités par an dont 1700 traductions).

\section{RÉSUMÉS}

Le tour d'horizon de la situation du catalan, en France, en tant que langue régionale (car il est langue co - officielle en Espagne et officielle en Andorre) a volontairement laissé de côté l'analyse des faits historiques largement débattus à ce jour, pour s'intéresser à l'impact de la mise en place de classes bilingues à parité horaire dans l'enseignement public dès 1993 et à la rédaction commencée en 2006 de programmes dans le cadre européen de référence qui permettent à la fois une harmonisation avec les autres langues vivantes et une remise en question des pratiques pédagogiques (approche actionnelle). Enfin, si l'enseignement - apprentissage des langues régionales est aujourd'hui perçu comme un «plus » par une large majorité de la population et a bien progressé dans les dix dernières années, le manque de moyens humains (professeurs) et financiers (dotations horaires) reste encore un handicap certain pour répondre aux besoins.

The general survey of the situation of Catalan in France, as a regional language (for it is co official in Spain and official in Andorra) has deliberately let aside the analysis of historical facts, some facts widely debated upon nowadays, to focus only on the impact of the enforcement of bilingual classes with equivalent schedule in public education, as soon as 1993 and on the wording, started in 2006, of European programmes within the Common European Framework of Reference for languages which allow on the one hand a harmonization with the other foreign languages and a questioning of practices (actional method). Finally, if the teaching - learning of regional languages is now considered as a bonus by a large majority of the population and has improved in the last ten years, the lack of human (teachers) and financial (teaching hours) resources undoudtedly remains a handicap to cope with the needs.

\section{INDEX}

Mots-clés : bilinguisme, cadre européen, perspective

Keywords : bilingualism, common european framework, prospect

\section{AUTEURS}

\section{MARY SANCHIZ}

I.A. - I.P.R. chargée de la mission catalan 


\section{LUC BONET}

Professeur formateur de catalan à l'IUFM de Perpignan 